\title{
The role of energy dissipation of polymeric scaffolds in the mechanobiological modulation of chondrogenic expression
}

\author{
Philippe Abdel-Sayed, Salim E. Darwiche, Ulrike Kettenberger, Dominique P. Pioletti*
}

Laboratory of Biomechanical Orthopedics, Institute of Bioengineering, EPF Lausanne, Switzerland

\section{A R T I C L E I N F O}

\section{Article history:}

Received 23 September 2013

Accepted 17 November 2013

Available online 9 December 2013

\section{Keywords:}

Cartilage

Dissipation

Chondrogenic expression

Mechanobiology

Scaffold

Dynamic compression

\begin{abstract}
A B S T R A C T
Mechanical stimulation has been proposed to induce chondrogenesis in cell-seeded scaffolds. However, the effects of mechanical stimuli on engineered cartilage may vary substantially between different scaffolds. This advocates for the need to identify an overarching mechanobiological variable. We hypothesize that energy dissipation of scaffolds subjected to dynamic loading may be used as a mechanobiology variable. The energy dissipation would furnish a general criterion to adjust the mechanical stimulation favoring chondrogenesis in scaffold. Epiphyseal chondro-progenitor cells were then subject to unconfined compression $2 \mathrm{~h}$ per day during four days in different scaffolds, which differ only by the level of dissipation they generated while keeping the same loading conditions. Scaffolds with higher dissipation levels upregulated the mRNA of chondrogenic markers. In contrast lower dissipation of scaffolds was associated with downregulation of chondrogenic markers. These results showed that energy dissipation could be considered as a mechanobiology variable in cartilage. This study also indicated that scaffolds with energy dissipation level close to the one of cartilage favors chondrogenic expression when dynamical loading is present.
\end{abstract}

(c) 2013 Elsevier Ltd. All rights reserved.

\section{Introduction}

Mechanical stimulation has been demonstrated to be one of the strategies for enhancing chondrogenesis and improving the mechanical properties of cell-based constructs [1-4]. Li and coworkers have shown that frequency and amplitude of dynamic compression modulate chondrogenesis of human bone marrow mesenchymal stem cells seeded in polymeric scaffolds [5]. However, specific frequency and amplitude may induce an effect in a particular scaffold that would be different in another. Indeed, the effects of mechanical stimuli on engineered cartilage may vary substantially between different scaffold types [6], probably because of different cell-scaffold interaction [7]. Consequently, interplay between dynamic compression and cell-matrix interaction may inhibit [8] or induce [9] the formation of cartilage-like tissues by chondrocytes. The conflicting results regarding the beneficial effect on chondrogenesis of a dynamic compression advocate to identify an overarching mechanobiological variable. This variable could then be used as a general criterion to adjust the mechanical stimulation favoring chondrogenesis in scaffold under mechanical stimulation.

\footnotetext{
* Corresponding author. Laboratory of Biomechanical Orthopedics, Institute of Bioengineering, Station 19, 1015 Lausanne, Switzerland. Tel.: +41 216938341

E-mail address: dominique.pioletti@epfl.ch (D.P. Pioletti).
}

Energy dissipation is a typical characteristic of viscoelastic materials such as polymeric scaffolds or articular cartilage [10]. Dissipation in cartilage relies on two internal mechanisms during deformation [11]. These mechanisms arise from the biphasic nature of articular cartilage, composed of a solid phase and liquid phase as modeled by Mow et al. [12]. The first internal dissipative mechanism, called intrinsic viscoelasticity, is due to solid-solid interactions in the cartilage extracellular matrix [13-16]. Those interactions are characterized by mechanical friction, chemical and electrostatic interactions, as well as physical entanglements between solid components of articular cartilage [14]. The second internal dissipative mechanism, called frictional drag, results from fluid-solid interactions. When articular cartilage is compressed, fluid movement occurs inducing frictions relative to the solid phase [12]. The two dissipative mechanisms may influence the cells behavior, either mediated by the glycocalix in response to fluid shear stress, or initiated by the force-induced unfolding of ECM proteins [17].

The dissipative properties of cartilage are usually characterized by hysteresis stress-strain curve [18]. In this work, we propose to consider energy dissipation as an overarching mechanobiological variable measured through a hysteresis curve because: (i) it encompasses all dissipative mechanisms related to solid and fluid phases, which may affect cell behavior; (ii) it is correlated to the tissue or scaffold microstructure (e.g. crosslinking and 


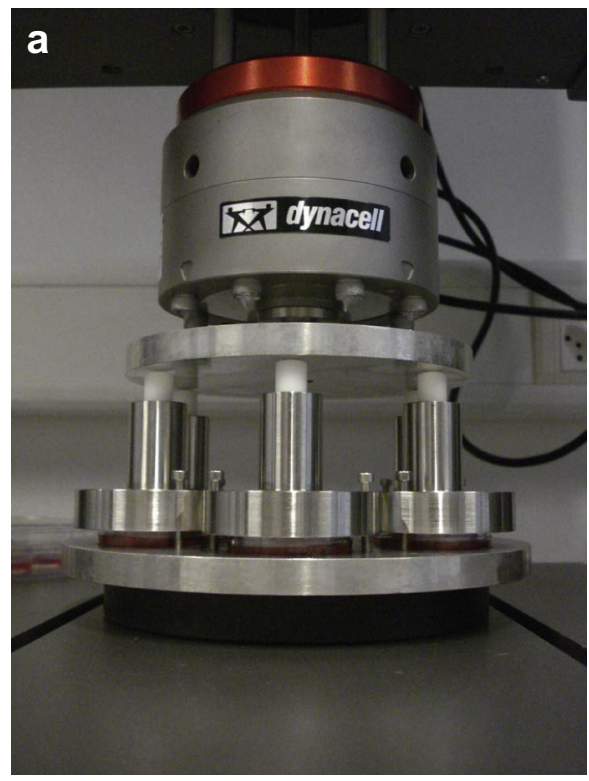

b

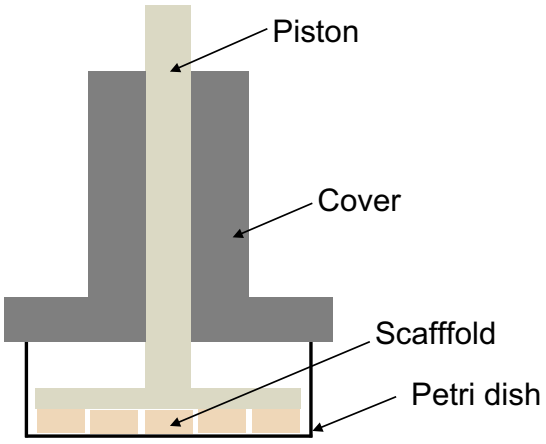

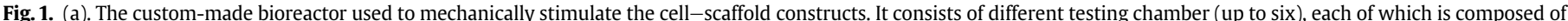

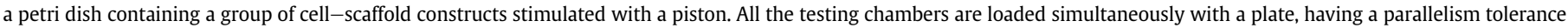

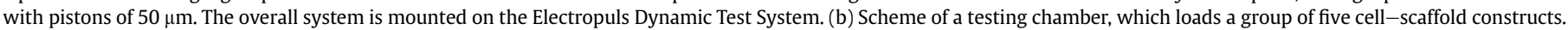
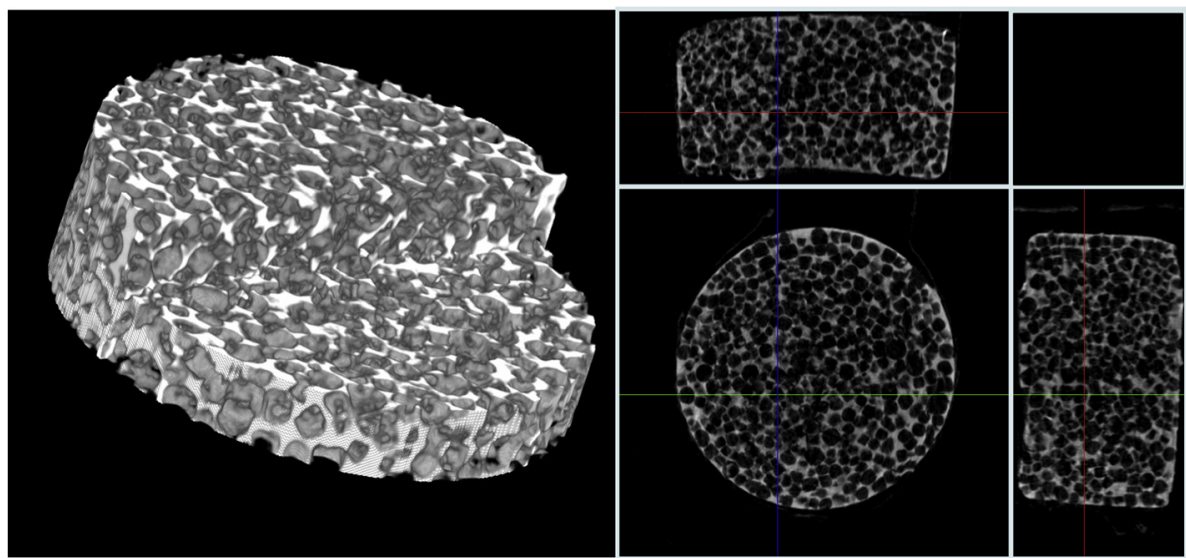

Fig. 2. Image of a reconstructed p(HEMA-co-EGDMA) scaffold from $\mu$ CT scans, showing the pores interconnectivity
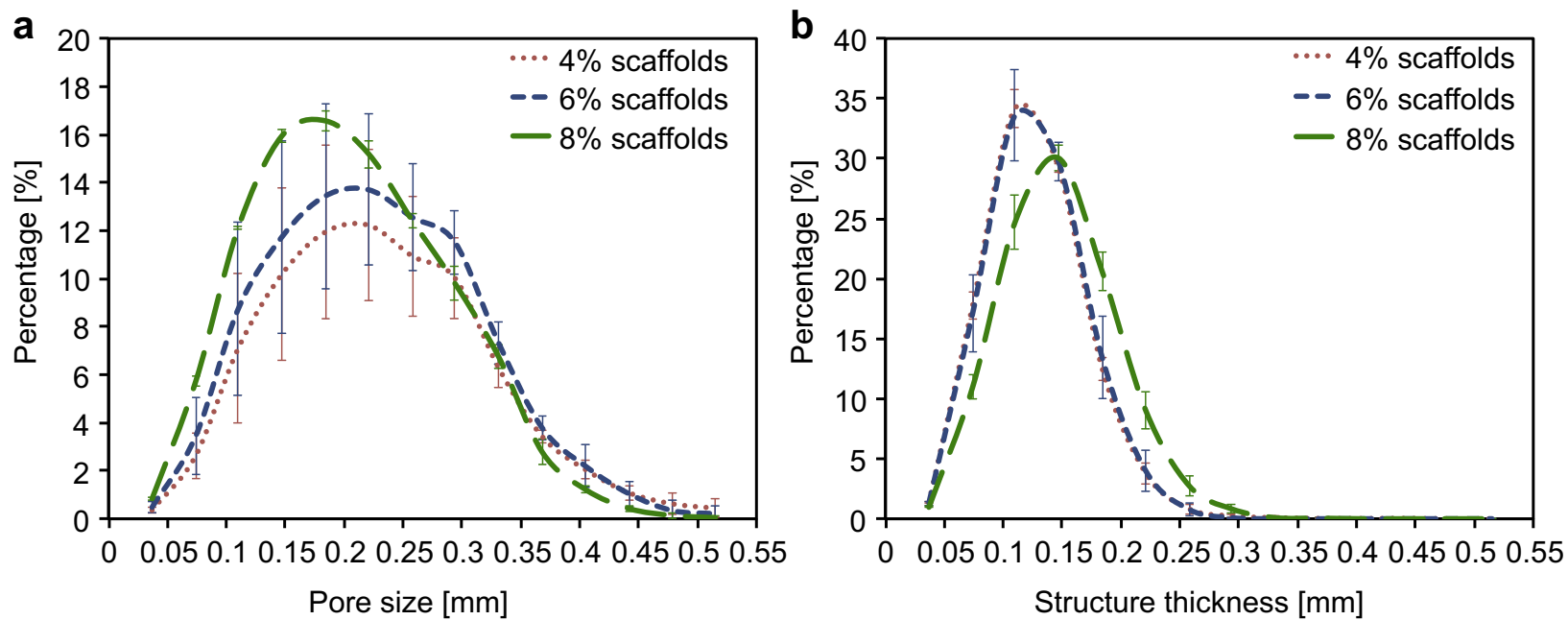

Fig. 3. Distribution of (a) pores size and (b) structure size in $\mathrm{p}$ (HEMA-co-EGDMA) scaffolds having $4 \%, 6 \%$ and $8 \%$ crosslinkers. For each group of scaffold $n=3$. 
entanglement); (iii) it is related to tissue or scaffold mechanical properties; and (iv) it is dependent on parameters of the mechanical stimulation, thus allowing to tune the dissipation level.

We hypothesize that chondrocyte behavior is sensitive to dissipation, having maximum chondrogenic expression at a dissipation level close to the one of normal healthy cartilage. The aim of the present study is thus to assess the effect of energy dissipation on chondrogenic expression of polymeric scaffolds under mechanical load.

\section{Material and methods}

2.1. Poly-(2-hydroxyethyl methacrylate-co-ethylene glycol dimethacrylate) ( $p($ HEMA-co-EGDMA)) scaffolds preparation

Different formulations of HEMA-based scaffolds were prepared in order to induce different levels of dissipation when subjected to the same loading in terms of frequency and amplitude of stimulation. The levels of dissipation were chosen to span the values between cartilage dissipations of healthy and degenerated samples. Scaffolds were prepared by a salt leaching method to obtain a macroporous structure. Briefly, $932 \mu$ of 2-hydroxyethyl methacrylate (HEMA) were mixed with $38 \mu \mathrm{l}$ ammonium persulfate water solution $(0.438 \mathrm{M}), 38 \mu \mathrm{l}$ sodium metabisulfite water solution $(0.526 \mathrm{~m})$ and with ethylene glycol dimethacrylate (EGDMA). This latter was used as a crosslinker and its percentage was calculated as mol $\mathrm{EGDMA}_{\mathrm{mol}} / \mathrm{mol}_{\mathrm{HEMA}}$. Solutions with $4 \%, 6 \%$, and $8 \%$ crosslinker were prepared and were poured in cylindrical molds ( $8 \mathrm{~mm}$ in diameter and $2 \mathrm{~mm}$ in height) containing salt and heated at $65{ }^{\circ} \mathrm{C}$ for $2 \mathrm{~h}$ to polymerize. The scaffolds were washed with bi-distilled water for $72 \mathrm{~h}$ in order to remove the salt, leaving a macroporous structure. The height was verified to be identical for all the scaffolds with a caliper having the precision of $\pm 10 \mu \mathrm{m}$. Scaffolds were autoclaved $20 \mathrm{~min}$ at $100{ }^{\circ} \mathrm{C}$ for sterilization. The aim of using different concentrations of crosslinker was to obtain different levels of dissipation given a same loading (same amplitude and frequency). The salt leaching technique was used to keep the same macroporous structure between the scaffolds that have different amounts of crosslinker, as previously shown [19]

\subsection{Structure of $p(H E M A-c o-E G D M A)$ scaffolds}

Scaffold morphologies were determined by micro-computed tomography (Skyscan 1076, Bruker-microCT, Kontich, Belgium). The measurement parameters were set as follows: spatial resolution $18 \mu \mathrm{m}$, voltage $40 \mathrm{kV}$, current $100 \mu \mathrm{A}$, exposure time $400 \mathrm{~ms}$ and rotation step $0.42^{\circ}$, without filter. The images were reconstructed using Nrecon software (Bruker-microCT). Structural parameters such as porosity, pore size and structure thickness (thickness of the cell walls) were obtained using CTAn software (Bruker-microCT). Distribution graphs were plotted to examine differences in morphologies between the scaffolds that have different amounts of crosslinker $(n=3)$.

\subsection{Scanning electron microscopy (SEM) of p(HEMA-co-EGDMA) scaffolds}

Surface topographic features including pores, pillars, or grooves influence cellular behavior [20]. SEM was used to verify that the topography is the same between the scaffolds having different amounts of crosslinker. Cross-sections (500$1000 \mu \mathrm{m}$ thick) of scaffold were dehydrated by cryo-lyophilization (freeze dryer Alpha 1-4, CHRIST GmbH, Osterode, Germany). The samples were then mounted on double-sided carbon tabs and coated with a $25 \mathrm{~nm}$ layer of gold/palladium. The samples were imaged using a XLF30-FEG instrument (FEI, Hillsboro, Oregon, USA) at an accelerating voltage of $5 \mathrm{kV}$, with a $3 \mathrm{~mm}$ working distance and an $11 \mu \mathrm{m}$ aperture.

\subsection{Mechanical properties of $p($ HEMA-co-EGDMA) scaffolds}

Energy dissipation and damping ratio of the scaffolds were quantified. Unconfined compression tests were made using an Electropuls Dynamic Test System (Instron E3000, Instron, Norwood, Massachusetts, USA) on cylindrical specimens immersed in deionized water at room temperature placed in a custom-made set-up. Prestrain of $10 \%$ was applied, followed by a sinusoidal compressive load of $10 \%$ amplitude at a frequency of $1 \mathrm{~Hz}$. The dissipation was calculated from the loaddisplacement graphs as the integral of the area enclosed by the hysteresis curve. The damping ratio was calculated as the energy dissipation divided by the total energy given to the system for maximum displacement. A $t$-test was used to examine the differences in dissipation between the scaffolds that have different concentrations of crosslinker $(n=4)$.

\subsection{Mechanical testing of cartilage samples}

We compared the dissipation of scaffolds with dissipation of cartilage. Hence, human cartilage samples were punched ( $8 \mathrm{~mm}$ diameter) from the femoral head of donors who have undergone hip arthroplasty (Centre Hospitalier Universitaire Vaudois Ethics Committee Protocol \# 264/12). The samples were taken from different sites, three samples were punched close to the site of degeneration
$(<2 \mathrm{~cm})$, and the others more than $2 \mathrm{~cm}$ further away. We defined each sample as "healthy" or "degenerated" depending on the their dynamic stiffness, as it has been shown that in osteoarthritic cartilage dynamic stiffness is $70 \%$ lower than in healthy cartilage [21]. Unconfined mechanical loading was performed as described previously for the scaffolds. The dynamic stiffness was measured as the slope between the maximum force-displacement and the minimum force-displacement.

\subsection{Human epiphyseal chondro-progenitors cells (ECPS) culture}

Human ECPs were isolated from the proximal ulnar epiphysis of a 14-week gestation donor (Centre Hospitalier Universitaire Vaudois Ethics Committee Protocol \#62/07). The isolation procedure was carried out according to our previous work [22]. Monolayer expansions were performed in standard tissue culture polystyrene flasks containing $10 \mathrm{ml}$ Dulbecco's Modified Eagle Medium (DMEM) with $25 \mathrm{~mm}$ dextrose and $1 \mathrm{~mm}$ sodium pyruvate (Life Technologies Ltd., Paisley, UK), $5.97 \mathrm{~mm} \mathrm{~L}-$
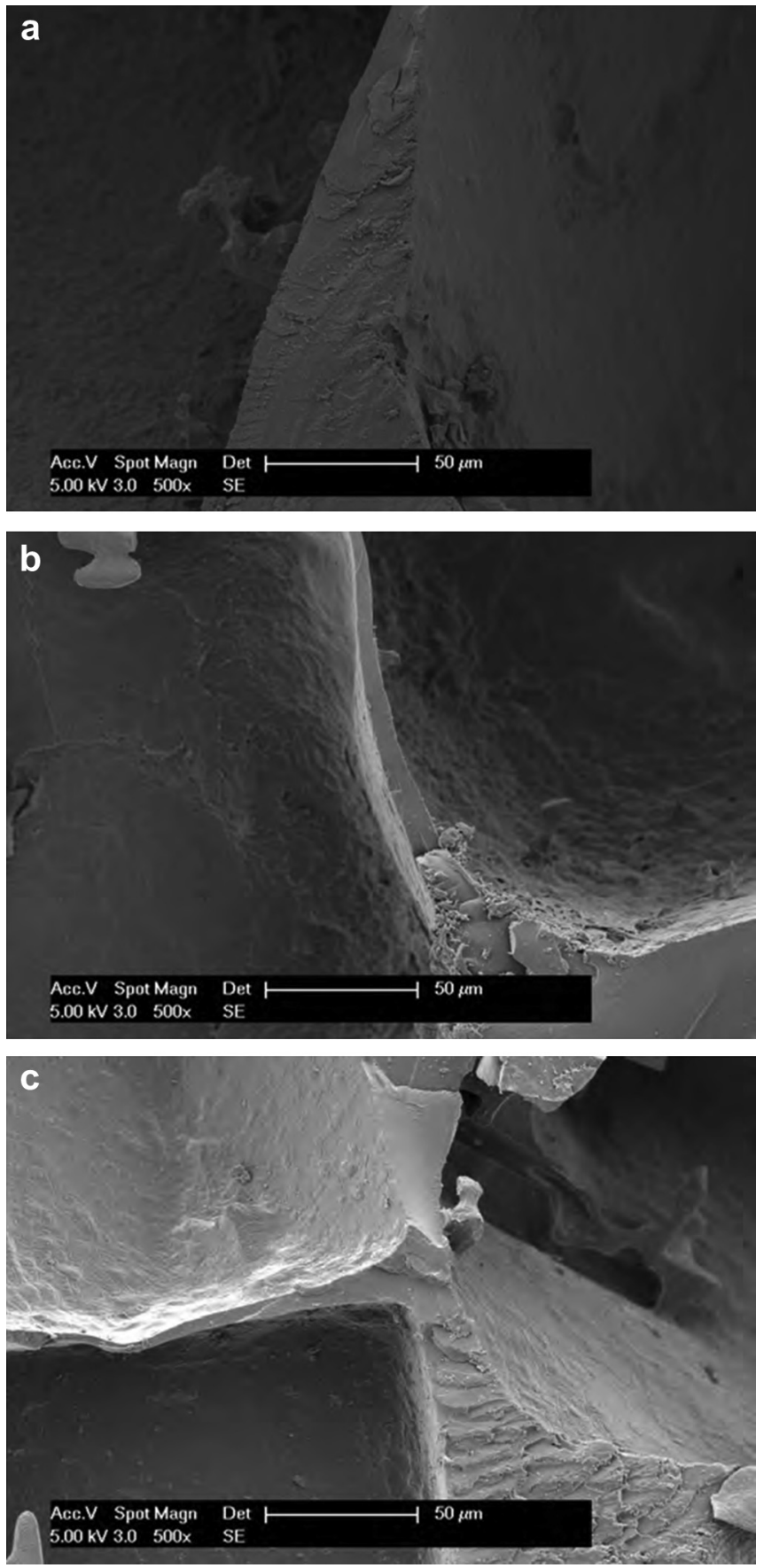

Fig. 4. SEM images of p(HEMA-Co-EGDMA) scaffolds having (a) $4 \%$, (b) $6 \%$, and (c) $8 \%$ crosslinker. 
glutamine (Life Technologies Ltd.), 10\% fetal bovine serum (Sigma, St. Louis, MO, USA). Human ECPs were seeded at 3300 cells $/ \mathrm{cm}^{2}$, placed in standard humidified tissue culture incubators at $37{ }^{\circ} \mathrm{C}$ with $5 \% \mathrm{CO}_{2}$ until reaching $90 \%$ confluence.

\subsection{Cell-scaffold constructs preparation}

Since different groups of scaffold having different amounts of crosslinker were considered, it was important to have identical cell-scaffold interface for the different groups, thus avoiding cellular behavior to be biased by different cellscaffold interfaces. Therefore all the scaffolds were coated with fibronectin to obtain a similar interface between the cells and the scaffold. For that purpose, scaffolds were placed in $2 \mathrm{ml}$ syringes and were coated by perfusing $300 \mu \mathrm{l}$ of a fibronectin solution $(10 \mu \mathrm{g} / \mathrm{ml}$, Sigma-Aldrich, St. Louis, Missouri, USA), and incubated $2 \mathrm{~h}$ at room temperature to allow adsorption of the fibronectin. Then the scaffolds were thoroughly washed with PBS and seeded with $300 \mu \mathrm{l}$ cell-solution resuspended in medium at a concentration of $3 \cdot 10^{6}$ cells $/ \mathrm{ml}$. Constructs were transferred to $35 \mathrm{~mm}$ Falcon ${ }^{\circledR}$ petri dishes (Becton Dickinson Labware, Franklin Lakes, New Jersey, USA) containing DMEM with $10 \%$ FBS, $1 \%$ L-glutamine and $1 \%$ PS for 4 days pre-incubation before mechanical testing.

\subsection{Cell viability and adhesion}

The LIVE/DEAD ${ }^{\circledR}$ Viability/Cytotoxicity staining kit (Invitrogen, Life Technologies Ltd., Paisley, UK) was used to qualitatively assess live and dead cells up to 28 days after seeding. Immunostaining of actin filament and fibronectin was used to verify cell adhesion on scaffolds after 4 days pre-culture just prior to the mechanica stimulation. Briefly, cell-seeded scaffolds were washed with PBS, fixed in $4 \%$ paraformaldehyde for $10 \mathrm{~min}$ at room temperature then washed thoroughly with PBS before proceeding to permeabilize cell membranes with a $0.2 \%$ solution of Triton-X in PBS for $20 \mathrm{~min}$ at room temperature. The permeabilizing solution was then washed with PBS before incubating in the dark for $1 \mathrm{~h}$ at room temperature in a solution containing rabbit anti-fibronectin (1:100, Sigma-Aldrich, St. Louis, Missouri, USA). This primary antibody was washed prior to incubation in the dark for $1 \mathrm{~h}$ with a secondary antibody (donkey anti-rabbit Alexa 568,1/800, Sigma-Aldrich, St. Louis, Missouri, USA) to stain fibronectin. In parallel to the secondary antibody, AlexaFluor $488^{\circledR}$ conjugated Phalloidin (1:100, Invitrogen, Life Technologies Ltd., Paisley, UK) was incubated in a $1 \%$ BSA solution in PBS to stain actin fibers. Samples were washed with PBS and cell staining was visualized using the Zeiss LSM 700 inverted confocal microscope (Carl Zeiss, Jena, Germany).

\subsection{Intermittent dynamic mechanical stimulation of cells}

After a preliminary culture of 4 days, half of the cell-scaffold constructs were subject to intermittent dynamic stimulation (loaded group) and half remained in free swelling (control group). Each group was constituted of scaffolds $(n=5)$ having respectively $4 \%, 6 \%$ and $8 \%$ crosslinker. The five constructs of each scaffold type were placed in a same testing chamber of a stimulation system developed in our laboratory (Fig. 1). All the scaffolds were subject to the same mechanical stimulus in parallel. An initial 10\% pre-strain was applied, followed by a sinusoidal compressive loading with $10 \%$ amplitude at a frequency of $1 \mathrm{~Hz}$ for $2 \mathrm{~h}$. After the unconfined compression test, specimens were unloaded and incubated for $22 \mathrm{~h}$ to allow constructs to recover. Experiments were conducted for 4 consecutive days. Constructs of the free-swelling group were subject to the same conditions except compression tests. Half of the culture medium was changed every day before the compression test for all the groups. At the fourth day of testing, immediately after mechanical stimulation gene expressions of the loaded and the free-swelling groups were analyzed.

\subsection{Gene expression}

Total RNA was extracted using NucleoSpin ${ }^{\circledR}$ RNA XS kit (Macherey-Nagel, Düren, Germany) according to the manufacturer's instructions. Prior to extraction, the scaffolds were milled using an Ultra-Turrax ${ }^{\circledR}$ disperser (IKA-WERK GmbH \& Co., Staufen, Germany). The RNA was quantified using Nanodrop ND-1000 Spectrophotometer (Witec AG, Pfäffikon, Switzerland), and 500 ng RNA was reversed transcribed into cDNA using Taqman ${ }^{\circledR}$ Reverse Transcription Reagents (Applied Biosystems, Life Technologies Ltd., Paisley, UK). Quantitative PCR was performed using the Fast SYBR ${ }^{\circledR}$ Green PCR Master Mix (Applied Biosystems) as well as primers synthesized by Microsynth (Balgach, Switzerland) for Sox9, Acan, Col2a1, TGF-beta3 and the housekeeping genes B2M (Table 1S, Supplementary data). Sox9, Col2a1, Acan and TGF-beta3 were chosen as biological targets to quantify how mechanical stimulation may regulate their expression $[3,23,24]$. They are essential targets, since they are markers of chondrogenesis expressed early during differentiation and are present throughout the different zones of cartilage [25]. Efficiency of the primers was determined using cDNA dilutions over 4 decades, and ranged from 89 to $110 \%$. Ten ng cDNA were added to each well of the reaction plate (MicroAmp Fast Optical 96-well, Applied Biosystems). The StepOnePlus Real-Time PCR system (Applied Biosystems) was used to perform the quantitative PCR. Gene expression data was processed following the comparative $\mathrm{Ct}$ method [26], normalizing gene expression levels by that of the housekeeping gene. For each scaffold group having the same crosslinker amount, the normalized gene expression level of the corresponding the free-swelling scaffolds was used as a biological reference. A $t$-test was used to examine the differences in gene expression results $(n=5)$.

\section{Results}

\section{1. $p($ HEMA-co-EGDMA) scaffolds}

Micro-computed tomography of the different scaffold groups showed interconnectivity between the pores (Fig. 2), with distributions of pore size and of structure thickness similar between groups of scaffold (Fig. 3). The mean values of all the scaffolds for pore size and structure thickness were $184 \mu \mathrm{m}$ and $140 \mu \mathrm{m}$ respectively. The mean porosity was $67 \%$ with $99 \%$ open pores. SEM images showed that the surface topography was similar between the different groups of scaffold, and no apparent micro-porosity was present (Fig. 4).

Dynamic stiffness of cartilage obtained less than $2 \mathrm{~cm}$ from the site of degeneration was $52 \pm 27 \mathrm{~N} / \mathrm{mm}$ while dynamic stiffness of the samples punched further away $(>2 \mathrm{~cm})$ was $171 \pm 45 \mathrm{~N} / \mathrm{mm}$. It can be concluded from these measurements that healthy and degenerated cartilages were obtained from the samples biopsy.
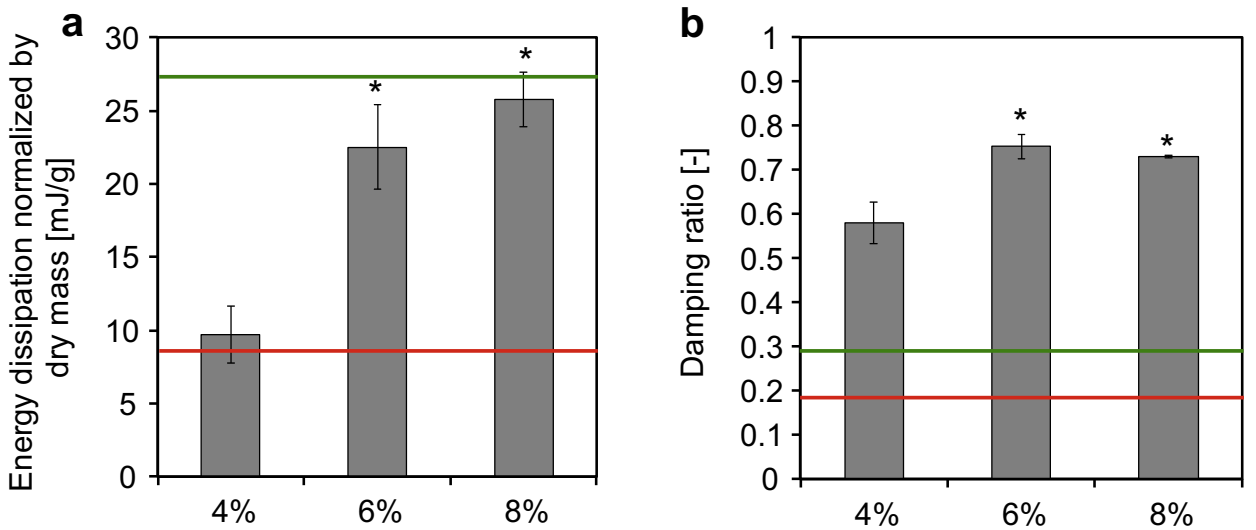

Percentage of crosslinker [mol $\left.\mathrm{mGDMA} / \mathrm{mol}_{\mathrm{HEMA}}\right]$ Percentage of crosslinker [mol $\mathrm{mGDMA}_{\mathrm{mol}} / \mathrm{mol}_{\mathrm{HEMA}}$ ]

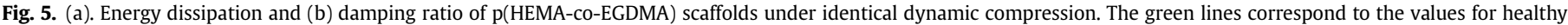

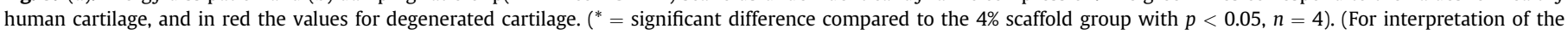
references to color in this figure legend, the reader is referred to the web version of this article.) 
Under an identical dynamic compression, the dissipation was higher for scaffolds with higher amount of crosslinker (Fig. 5.a). The dissipation of the $8 \%$ scaffold group was close to the dissipation of healthy cartilage. Damping ratios were around 0.7 except for the $4 \%$ scaffold group, which was around 0.6. The damping ratios were much higher in all the scaffolds compared to the damping ratios of cartilage (Fig. 5.b).

\subsection{Cell viability and adhesion}

Cell viability after 28 days was good for all the different groups as shown in Fig. 6. Cell adhesion on scaffolds was verified by immunohistochemistry performed on fibronectin-coated scaffolds and on non-coated scaffolds as control. For those latter, fibronectin deposed by cells and cells actin fibers may be observed in Fig. 7. It can be noticed that cells do not secret fibronectin on scaffolds with low percentage of crosslinker, while they produce more fibronectin on scaffolds with higher percentage of crosslinker. Therefore, without coating the cell-scaffold interface is not the same between the different groups of scaffold. Immunohistochemistry performed on fibronectin-coated scaffolds showed homogeneous distribution of the fibronectin allover the scaffold surface. A stretching of the actin fibers was also observed, which is a sign of a good homogeneous adhesion of the cells to the scaffold surface (Fig. 7).

\subsection{Modulation of chondrogenic expression by energy dissipation of scaffolds}

The chondrogenic expression profiles for cell-scaffold constructs presenting different dissipation are reported in Fig. 8. In general, an upregulation is observed for all the genes with an increase of scaffold dissipation. Indeed, the $8 \%$ scaffolds were those that had the closest dissipation level to the dissipation of healthy cartilage, and it is also the group of scaffolds for which maximal mRNA level was observed for TGF-beta3, Sox9, Acan, and Col2a1. The $4 \%$ scaffolds were those that had the closest dissipation level to the dissipation of degenerated cartilage, and it is also the group of scaffolds for which minimal mRNA level was observed for Sox9, Acan, and Col2a1. Downregulation was even observed in $4 \%$ scaffolds for Acan compared to the free-swelling group.

\section{Discussion}

Cartilage displays viscoelastic behavior [13,15,27-33] that arises from the complex structure and interactions of its biochemical constituents: mostly water, electrolytes, and a solid matrix composed primarily of collagen and proteoglycans [34]. A characteristic of viscoelasticity is the energy dissipation during dynamic stimulation. The goal of this study was to evaluate dissipation as mechanobiological variable affecting chondrogenic expression. Thus, scaffolds dissipating differently under identical loading and having the same structure and cell-scaffold interface were developed. The scaffolds were seeded with epiphyseal chondroprogenitors cells and were subject to unconfined compression 2 h/day during 4 days. In general, higher dissipation close to dissipation of healthy cartilage had maximal mRNA level for chondrogenic markers, while scaffolds with lowest dissipation close to the dissipation of degenerated cartilage had minimal mRNA level for chondrogenic markers.

Some studies have shown the beneficial effect of dynamic unconfined compression on chondrogenesis, such as increase of GAGs deposition by mesenchymal stem cells [9,35], upregulation of chondrogenic markers and signaling receptors $[3,36]$. However, none of these studies has directly related the chondrogenic
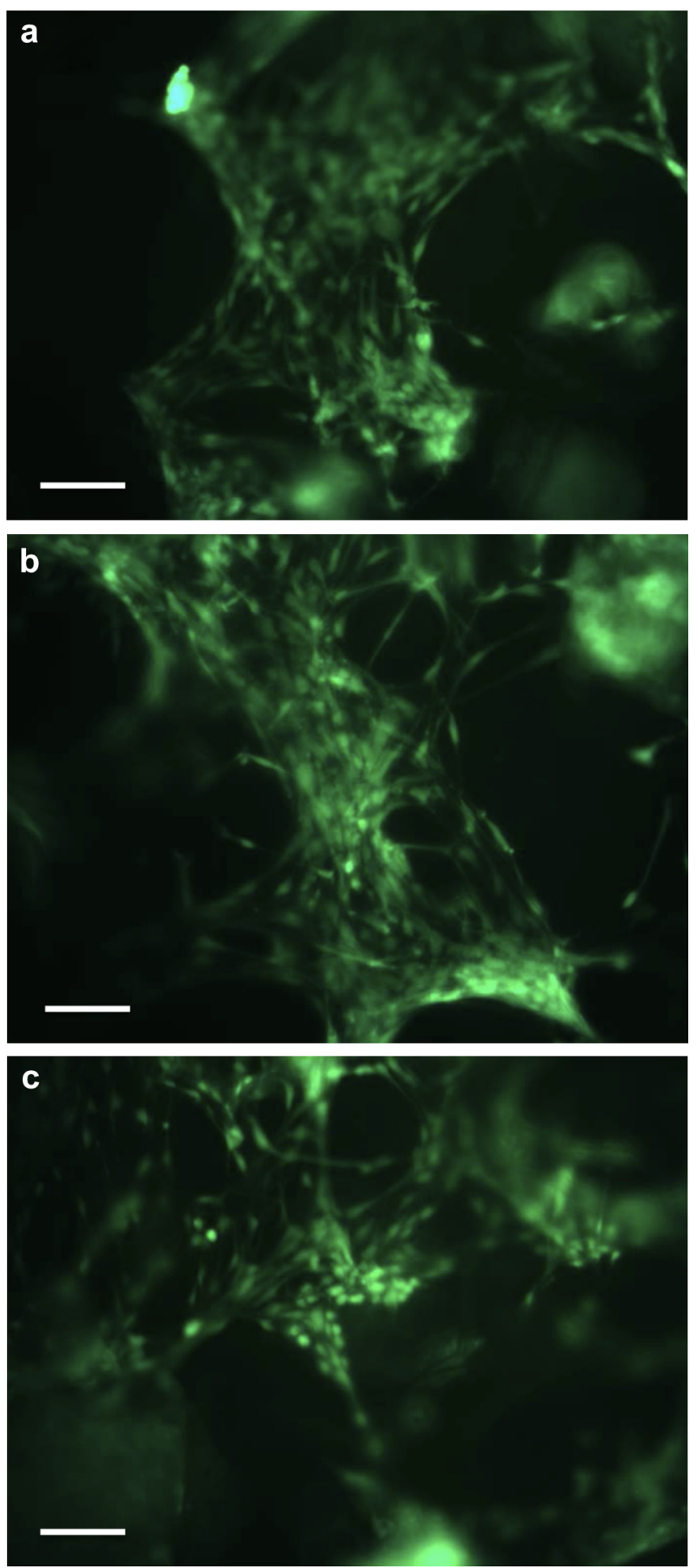

Fig. 6. Cell viability of epiphyseal chondro-progenitors cells in p(HEMA-co-EGDMA) scaffolds having (a) 4\%, (b) $6 \%$, and (c) $8 \%$ crosslinkers (scale bar $=100 \mu \mathrm{m}$ ).

response to dissipation. Nevertheless, some works have reported indirectly the effect of dissipative phenomena such as the influence of pressure variations due to fluid flow in the synthesis of matrix in engineered cartilage [37]. Indeed, fluid flow is one of the mechanisms that affect chondrocytes behavior [38]. The relevance of using hysteresis curve as a mechanobiological variable lays in the fact that it encompasses not only the dissipative effects linked to fluid 
(-) Fibronectin coated
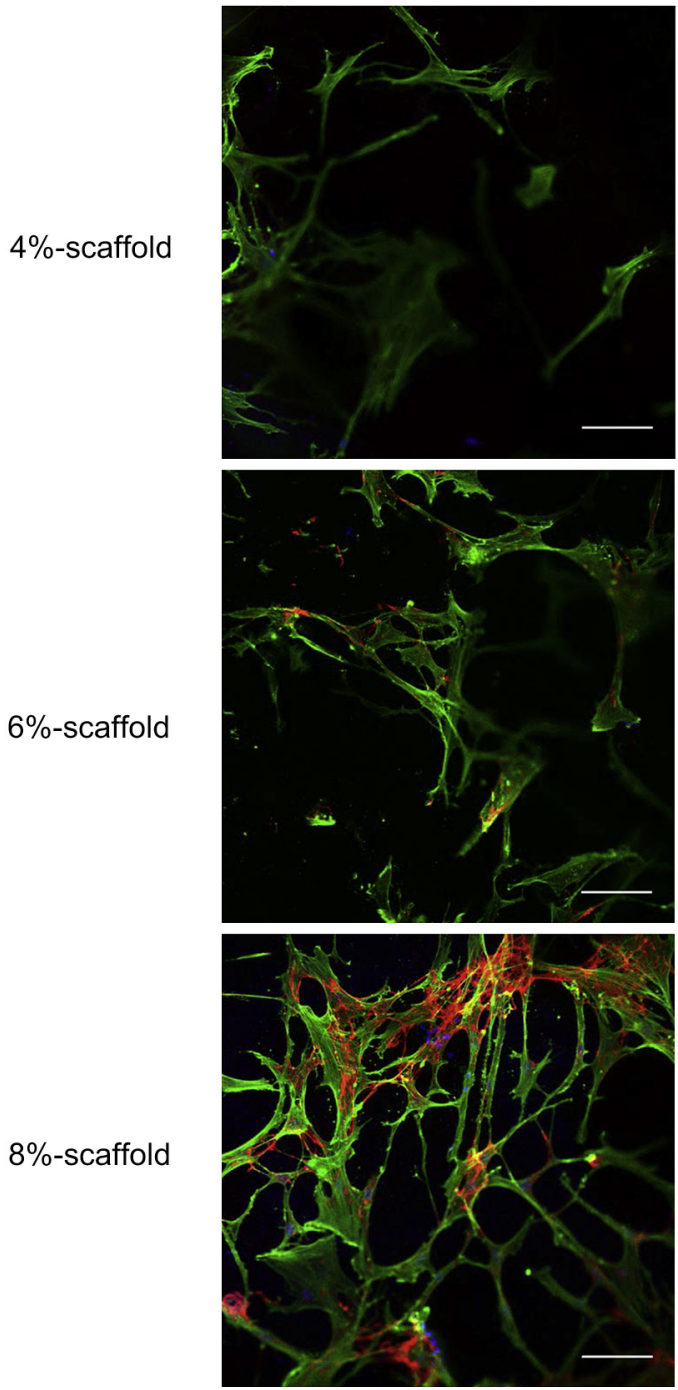

(+) Fibronectin coated
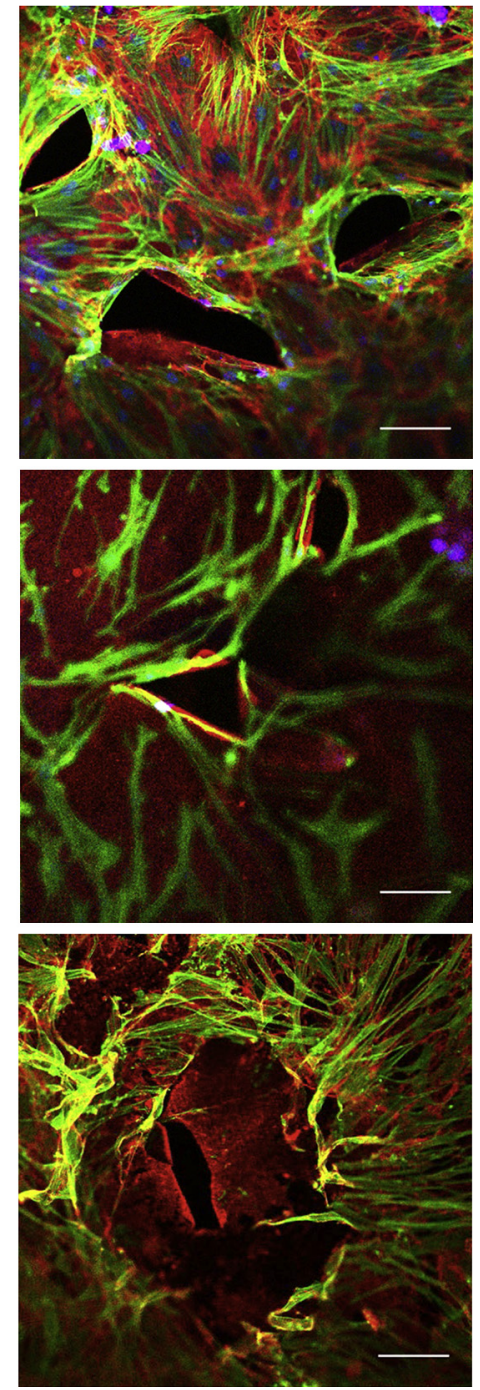

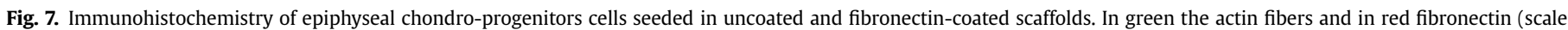
bar $=100 \mu \mathrm{m}$ ). (For interpretation of the references to color in this figure legend, the reader is referred to the web version of this article.)

flow phenomena, but also those of the solid matrix, which can as well induce mechanotransduction.

The suggestion of dissipation as a variable in mechanobiology has already been proposed for bone. Indeed, in vitro deformation of neonatal mouse bone at different frequencies and amplitudes has shown greatest anabolic responses at maximal energy dissipation [39]. Likewise, a model has been proposed for bone adaptation based on cyclic energy dissipation as measure of bone damage creation [40]. This formulation based on dissipation gave more accurate predictions than the ones given by the strain energy based adaptation theories. Actually, the latter consider tensile and compressive strains identically, while dissipative formulations gave a better fitting of the experimental data in tension, which were different than in compression [41].

In this study, we observed upregulations that were in the range of 2- to 3-fold increase in mRNA expression for Sox9 and Acan. This range is similar to the upregulations for same genes in mesenchymal progenitor cells dynamically stimulated without any external addition of growth factors [4]. The 22-fold increase in Col2a1 mRNA level showed the clear beneficial effect of higher dissipation on chondrogenic expression (Fig. 8.d). In our study, TGF- beta3 mRNA level was also upregulated subsequent to cyclic compression, indicating that the chondrogenic marker alteration may have been through the TGF-beta pathway, as previously observed in other studies [23,36].

We measured in this study, not only the value of scaffold energy dissipation, but also their damping ratio. Significant difference of the $6 \%$ and $8 \%$ scaffold compared to the $4 \%$ scaffold was associated with a difference in chondrogenic expression, sustaining the fact that a higher viscous component induces higher cellular effects. This viscous component in cartilage is governed by the proteoglycans. Indeed, digestion and removal of proteoglycans alone with cathepsin D caused the damping coefficient to decrease with no change in elastic stiffness [42,43]. Interestingly, it is also the proteoglycans, namely heparan sulfate, which mediate interstitial flow mechanotransduction $[44,45]$. Thus, the assumption of a viscous dissipation effect in cartilage mechanobiology is also supported by the role of proteoglycans to simultaneously govern viscosity and cartilage mechanobiology.

We have shown in this study that dissipation levels close to cartilage dissipation induced the highest chondrogenic expression of mechanically stimulated cell-seeded scaffolds, suggesting 


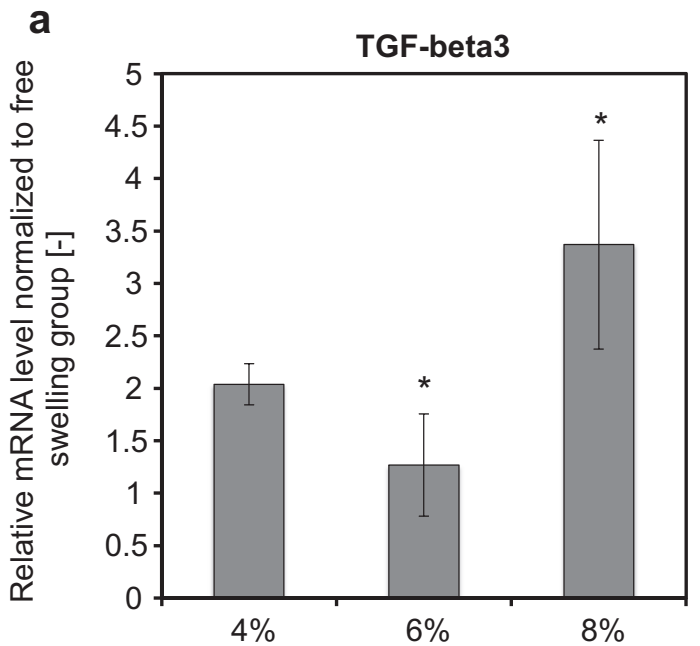

a

Scaffolds with different concentrations of crosslinker [mol EGDMA $/ \mathrm{mol}_{\text {HEMA }}$ ]

C

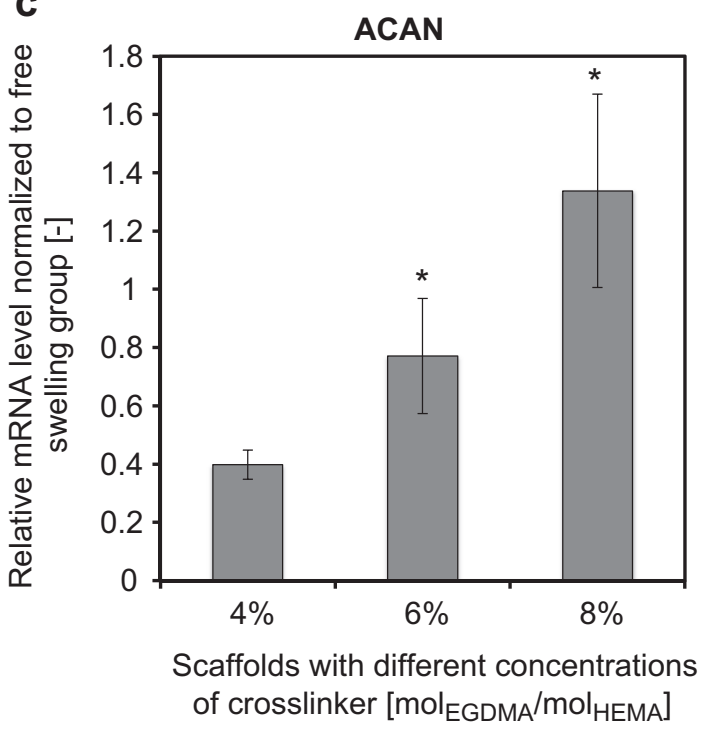

b

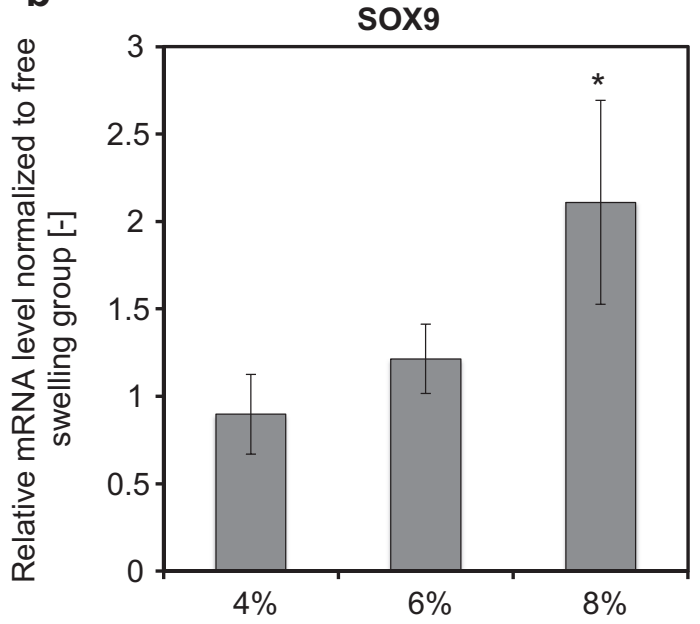

Scaffolds with different concentrations of crosslinker [mol EGDMA $_{\text {mol }} / \mathrm{m}_{\mathrm{HEMA}}$ ]

d
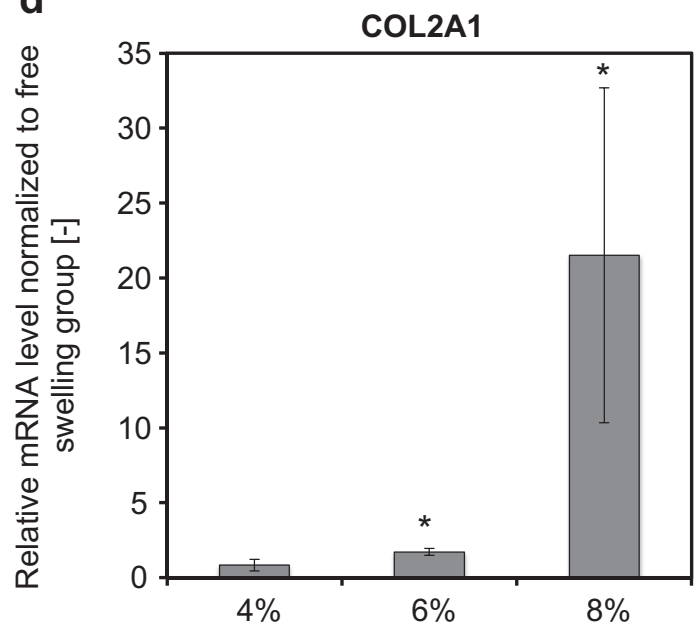

Scaffolds with different concentrations of crosslinker [mol $\left.\mathrm{mGDMA}_{\mathrm{mol}} / \mathrm{meMA}_{\mathrm{HE}}\right]$

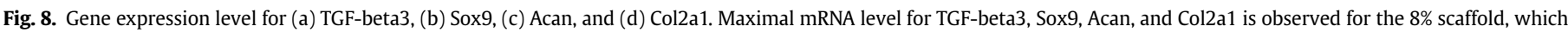

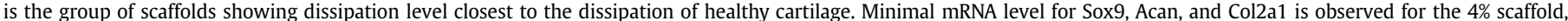

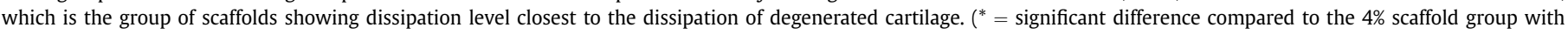
$p<0.05, n=5$ )

dissipation as a variable to tune the mechanical stimulation and to design scaffolds.

\section{Conclusions}

The goal of this study was to evaluate the effect of dissipation on chondrogenic expression. We developed p(HEMA-co-EGDMA) scaffolds having different levels of dissipation for an identical dynamic deformation. On a cellular level, we showed that chondrogenic expression of human chondro-progenitors cells was sensitive to dissipation. Higher dissipation levels close to cartilage dissipation upregulated the mRNA of chondrogenic markers. In contrast lower dissipation of scaffolds was associated with downregulation of chondrogenic markers. These results showed that energy dissipation could be involved in cartilage mechanobiology and that scaffold with energy dissipation value close to the one of healthy cartilage dissipation favors chondrogenesis when dynamical loading is present.

\section{Acknowledgments}

We would like to thank Sandra Jaccoud for technical assistance for cell culture. We also acknowledge the funding from the Swiss National Science Foundation (\#315230_125344) and the Interinstitutional Center for Translational Biomechanics EPFL-CHUVDAL.

\section{Appendix A. Supplementary data}

Supplementary data related to this article can be found at http:// dx.doi.org/10.1016/j.biomaterials.2013.11.048.

\section{References}

[1] Huang AH, Farrell MJ, Kim M, Mauck RL. Long-term dynamic loading improves the mechanical properties of chondrogenic mesenchymal stem cell-laden hydrogel. Eur Cell Mater 2010;19:72-85. 
[2] Campbell JJ, Lee DA, Bader DL. Dynamic compressive strain influences chondrogenic gene expression in human mesenchymal stem cells. Biorheology 2006;43(3-4):455-70.

[3] Huang CY, Reuben PM, Cheung HS. Temporal expression patterns and corresponding protein inductions of early responsive genes in rabbit bone marrowderived mesenchymal stem cells under cyclic compressive loading. Stem Cells $2005 ; 23(8): 1113-21$

[4] Terraciano V, Hwang N, Moroni L, Park HB, Zhang Z, Mizrahi J, et al. Differential response of adult and embryonic mesenchymal progenitor cells to mechanical compression in hydrogels. Stem Cells 2007;25(11):2730-8.

[5] Li Z, Yao SJ, Alini M, Stoddart MJ. Chondrogenesis of human bone marrow mesenchymal stem cells in fibrin-polyurethane composites is modulated by frequency and amplitude of dynamic compression and shear stress. Tissue Eng Pt A 2010;16(2):575-84.

[6] Hunter C, Mouw JK, Levenston ME. Dynamic compression of chondrocyteseeded fibrin gels: effects on matrix accumulation and mechanical stiffness. Osteoarthr Cartil 2004;12(2):117-30.

[7] Connelly JT, Petrie TA, Garcì AJ, Levenston ME. Fibronectin-and collagenmimetic ligands regulate bone marrow stromal cell chondrogenesis in three-dimensional hydrogels. Eur Cell Mater 2011:22:168-77.

[8] Thorpe SD, Buckley CT, Vinardell T, O’Brien FJ, Campbell VA, Kelly DJ. Dynamic compression can inhibit chondrogenesis of mesenchymal stem cells. Biochem Biophys Res Commun 2008;377(2):458-62.

[9] Mauck RL, Byers BA, Yuan X, Tuan RS. Regulation of cartilaginous ECM gene transcription by chondrocytes and MSCs in 3D culture in response to dynamic loading. Biomech Model Mechanobiol 2007;6(1-2):113-25.

[10] Shaw MT, MacKnight WJ. Introduction to polymer viscoelasticity. Wiley; 2005.

[11] Desmarais M, Aissaoui R. Modeling of knee articular cartilage dissipation during gait analysis. J Mech Med Biol 2008;8(3):377-94.

[12] Mow VC, Kuei SC, Lai WM, Armstrong CG. Biphasic creep and stress relaxation of articular cartilage in compression? Theory and experiments. J Biomech Eng 1980;102(1):73-84

[13] Zhu W, Mow VC, Koob TJ, Eyre DR. Viscoelastic shear properties of articular cartilage and the effects of glycosidase treatments. J Orthop Res 1993;11(6): $771-81$.

[14] Setton LA, Zhu WB, Mow VC. The biphasic poroviscoelastic behavior of articular-cartilage - role of the surface zone in governing the compressive behavior. J Biomech 1993;26(4-5):581-92.

[15] Hayes WC, Bodine AJ. Flow-independent viscoelastic properties of articularcartilage matrix. J Biomech 1978;11(8-9):407-19.

[16] Huang CY, Mow VC, Ateshian GA. The role of flow-independent viscoelasticity in the biphasic tensile and compressive responses of articular cartilage. J Biomech Eng 2001;123(5):410-7.

[17] Jaalouk DE, Lammerding J. Mechanotransduction gone awry. Nat Rev Mol Cell Biol 2009;10(1):63-73.

[18] Szarko M, Muldrew K, Bertram JEA. Freeze-thaw treatment effects on the dynamic mechanical properties of articular cartilage. BMC Musculoskel Disord 2010:11.

[19] Ghosh S, Gutierrez V, Fernandez C, Rodriguez-Perez MA, Viana JC, Reis RL, et al. Dynamic mechanical behavior of starch-based scaffolds in dry and physiologically simulated conditions: effect of porosity and pore size. Acta Biomater 2008;4(4):950-9.

[20] Vonrecum AF, Vankooten TG. The influence of micro-topography on cellularresponse and the implications for silicone implants. J Biomater Sci Polym Ed 1995:7(2):181-98.

[21] Knecht S, Vanwanseele B, Stussi E. A review on the mechanical quality of articular cartilage - implications for the diagnosis of osteoarthritis. Clin Biomech 2006;21(10):999-1012.

[22] Darwiche SE, Scaletta C, Raffoul W, Pioletti DP, Applegate LA. Epiphyseal chondroprogenitors provide a stable cell source for cartilage cell therapy. Cell Med 2012;4:23-32.
[23] Li Z, Kupcsik L, Yao SJ, Alini M, Stoddart MJ. Mechanical load modulates chondrogenesis of human mesenchymal stem cells through the TGF-beta pathway. J Cell Mol Med 2010;14(6):1338-46.

[24] Huang AH, Farrell MJ, Mauck RL. Mechanics and mechanobiology of mesenchymal stem cell-based engineered cartilage. J Biomech 2010;43(1):128-36.

[25] Zuscik MJ, Hilton MJ, Zhang XP, Chen D, O'Keefe RJ. Regulation of chondrogenesis and chondrocyte differentiation by stress. J Clin Invest 2008;118(2): 429-38.

[26] Pfaffl MW. A new mathematical model for relative quantification in real-time RT-PCR. Nucleic Acids Res 2001;29(9):e45.

[27] Fulcher GR, Hukins DWL, Shepherd DET. Viscoelastic properties of bovine articular cartilage attached to subchondral bone at high frequencies. BMC Musculoskel Disord 2009:10.

[28] Guilak F. The deformation behavior and viscoelastic properties of chondrocytes in articular cartilage. Biorheology 2000;37(1-2):27-44.

[29] Spirt AA, Mak AF, Wassell RP. Nonlinear viscoelastic properties of articularcartilage in shear. J Orthop Res 1989;7(1):43-9.

[30] Woo SLY, Simon BR, Kuei SC, Akeson WH. Quasilinear viscoelastic properties of normal articular-cartilage. J Biomech Eng 1980;102(2):85-90.

[31] Uchida S, Morita M, Fujimasu H. Frictional behavior and viscoelastic properties of articular-cartilage. J Jpn Soc Lubr Eng 1978;23(2):117-23.

[32] Repo RU, Evans JH, Barbenel JC. Effect of protein polysaccharide degradation on viscoelastic properties of human articular-cartilage. J Bone Joint Surg Am 1974;A 56(4):852.

[33] Hayes WC, Mockros LF. Viscoelastic properties of human articular cartilage. J Appl Physiol 1971;31(4):562.

[34] Cohen NP, Foster RJ, Mow VC. Composition and dynamics of articular cartilage: structure, function, and maintaining healthy state. J Orthop Sport Phys $1998 ; 28(4): 203-15$

[35] Mauck RL, Soltz MA, Wang CCB, Wong DD, Chao PHG, Valhmu WB, et al Functional tissue engineering of articular cartilage through dynamic loading of chondrocyte-seeded agarose gels. J Biomech Eng 2000;122(3):252-60.

[36] Mouw JK, Connelly JT, Wilson CG, Michael KE, Levenston ME. Dynamic compression regulates the expression and synthesis of chondrocyte-specific matrix molecules in bone marrow stromal cells. Stem Cells 2007;25(3): 655-63.

[37] Hunter CJ, Levenston ME. The influence of repair tissue maturation on the response to oscillatory compression in a cartilage defect repair model. Biorheology 2002;39(1-2):79-88.

[38] Degala S, Williams R, Zipfel W, Bonassar LJ. Calcium signaling in response to fluid flow by chondrocytes in 3D alginate culture. J Orthop Res 2012;30(5): 793-9.

[39] Kunnel JG, Gilbert JL, Stern PH. In vitro mechanical and cellular responses of neonatal mouse bones to loading using a novel micromechanical-testing device. Calcif Tissue Int 2002:71(6):499-507.

[40] Levenston ME, Carter D. An energy dissipation-based model for damage stimulated bone adaptation. J Biomech 1998;31(7):579-86.

[41] Pattin CA, Caler WE, Carter DR. Cyclic mechanical property degradation during fatigue loading of cortical bone. J Biomech 1996;29(1):69-79.

[42] Bader DL, Kempson GE. The short-term compressive properties of adult human articular cartilage. Biomed Mater Eng 1994;4(3):245-56.

[43] Bader DL, Kempson GE, Egan J, Gilbey W, Barrett AJ. The effects of selective matrix degradation on the short-term compressive properties of adult human articular cartilage. Biochim Biophys Acta 1992;1116(2):147-54.

[44] Shi ZD, Wang H, Tarbell JM. Heparan sulfate proteoglycans mediate interstitial flow mechanotransduction regulating MMP-13 expression and cell motility via FAK-ERK in 3D collagen. PLoS One 2011;6(1):e15956.

[45] Moon JJ, Matsumoto M, Patel S, Lee L, Guan JL, Li S. Role of cell surface heparan sulfate proteoglycans in endothelial cell migration and mechanotransduction. J Cell Physiol 2005;203(1):166-76. 